\title{
Procollagen-lysine, 2-oxoglutarate 5-dioxygenase 1 increases cellular proliferation and colony formation capacity in lung cancer via activation of E2F transcription factor 1
}

\author{
BAIJUN LI ${ }^{1 *}$, HAO YANG ${ }^{2 *}$, BIN SHEN $^{1}$, JIANWEI HUANG $^{1}$ and ZHIQIANG QIN ${ }^{3}$ \\ Departments of ${ }^{1}$ Thoracic Cardiovascular Surgery, ${ }^{2}$ Administration and ${ }^{3}$ Pulmonary and Critical Care Medicine, \\ The People's Hospital of Guangxi Zhuang Autonomous Region, Nanning, Guangxi 530021, P.R. China
}

Received July 24, 2020; Accepted May 25, 2021

DOI: $10.3892 / 01.2021 .13112$

\begin{abstract}
Procollagen-lysine, 2-oxoglutarate 5-dioxygenase 1 (PLOD1) is an enzyme that catalyzes the hydroxylation of lysyl residues in collagen-like peptides, and is responsible for the stability of intermolecular crosslinks. High PLOD1 mRNA levels have been determined to be prognostically significant in numerous human malignancies. The objective of the present study was to elucidate the pathological mechanism of PLOD1 in lung cancer. The expression status and prognostic value of PLOD1 in lung adenocarcinoma (LUAD) and lung squamous cell carcinoma (LUSA) were investigated using Gene Expression Profiling Interactive Analysis (GEPIA). Cell Counting Kit 8 and colony formation assays were performed to assess the impact of PLOD1 depletion and overexpression on the proliferation and colony formation abilities of the A549 lung cancer cell line. Luciferase reporter assays were used to clarify whether E2F transcription factor 1 (E2F1) was a downstream target of PLOD1 in lung cancer. Finally, the correlations between PLOD1 expression and a typical central downstream effector molecule of E2F1 signaling were determined using cBioportal. The GEPIA datasets revealed that PLOD1 mRNA levels were upregulated in LUAD and LUSC samples. Furthermore, the overexpression of PLOD1 promoted cancer cell proliferation and colony formation in vitro, while PLOD1-knockout produced the opposite effect. Notably, PLOD1 markedly induced the transcriptional activity of E2F1. Additionally, the expression of PLOD1 was significantly
\end{abstract}

Correspondence to: Professor Zhiqiang Qin, Department of Pulmonary and Critical Care Medicine, The People's Hospital of Guangxi Zhuang Autonomous Region, 6 Taoyuan Road, Nanning, Guangxi 530021, P.R. China

E-mail: qinzhiqiang148@sina.com

${ }^{*}$ Contributed equally

Key words: lung cancer, procollagen-lysine, 2-oxoglutarate 5-dioxygenase 1, E2F transcription factor 1, proliferation, colony formation correlated with that of $\mathrm{H} 2 \mathrm{~A}$ histone family member $\mathrm{X}$. In conclusion, the findings of the present study indicate that PLOD1 promoted lung cancer through E2F1 activation, and proposed a rationale for targeting the PLOD1/E2F1 axis to treat lung cancer.

\section{Introduction}

Lung cancer is one of the most frequently diagnosed cancers worldwide (1), with 2.1 million new cases reported in 2018, and is a major cause of cancer-related death (2) due to its highly aggressive nature and potential for metastasis (3). Despite recent advances in diagnosis and treatment, the prognosis of lung cancer remains poor (4). In particular, patients with clinical stage IV lung cancer have a 5-year overall survival (OS) rate of $2 \%$, and even for the lowest stage, IA, the 5-year OS rate is only 50\% (5). Therefore, achieving an improved understanding of the molecular pathogenesis of lung tumor progression may contribute to the development of early detection and targeted therapy.

The procollagen-lysine, 2-oxoglutarate 5-dioxygenases (PLODs), which catalyze lysyl hydroxylation to form hydroxylysine, comprise a group of enzymes that regulate extracellular collagen crosslinking and deposition, which is critical for extracellular matrix assembly and remodeling (6). High expression levels of PLODs have been observed in various cancer types, and have been found to regulate tumor growth, invasion and metastasis, as well as radio-resistance $(7,8)$. Furthermore, several studies have verified that certain clinicopathological characteristics that indicate a poor cancer prognosis are associated with PLOD expression, suggesting that PLODs are druggable therapeutic targets for cancer treatment $(9,10)$. PLOD1, a member of the PLOD family, also possesses multiple biological functions for the stabilization of collagen. Regarding cancer development, PLOD1 is defined as a positive tumor hypoxia gene and is correlated with distant metastasis, recurrence and unfavorable clinical outcomes in nine different cancer types, including lung adenocarcinoma (LUAD) $(8,10)$. Both small interfering RNA-PLOD1 and PLOD1-specific inhibitors resulted in reduced bladder cancer cell invasiveness and impeded bladder tumor progression (11). Notably, PLOD1 restoration can abrogate microRNA-induced 
suppression of osteosarcoma cell proliferation and aggressiveness via inactivation of the Hippo-YAP signaling pathway (12). However, the mechanisms of PLOD1 in lung cancer have not been fully elucidated.

E2F transcription factor 1 (E2F1) is a member of the E2F transcription factor family $(13,14)$ and is a central player governing cellular proliferation and metastatic survival of numerous cancer types, including lung cancer $(15,16)$. A decrease in E2F1 is reportedly involved in mitochondrial function and oxidative stress in lung cancer (17). PLOD1 expression has also been associated with hypoxic microenvironments in several types of cancer (15). Therefore, it is speculated that PLOD1 may regulate E2F1 to interfere with lung cancer progression.

The aim of the present study was to investigate the expression status of PLOD1 in LUAD and lung squamous cell carcinoma (LUSC) using a Gene Expression Profiling Interactive Analysis (GEPIA) dataset, and to determine the association between PLOD1 expression and survival. The effect of PLOD1 expression on lung cancer cell behavior was also evaluated, and the regulatory relationship between PLOD1 and E2F1 analyzed. The results may help to establish a theoretical basis for the PLOD1-targeted treatment of lung cancer.

\section{Materials and methods}

Cell culture. The human lung cancer cell lines A549 and NCI-H1975, and human 293 T cells, were originally obtained from the Chinese Academy of Sciences in Shanghai. All cells were cultured in DMEM supplemented with $10 \%$ fetal bovine serum and $1 \% 100 \mathrm{U} / \mathrm{ml}$ penicillin/streptomycin, and maintained at $37^{\circ} \mathrm{C}\left(5 \% \mathrm{CO}_{2}\right)$ in a humidified incubator.

Reverse transcription-quantitative $(R T-q) P C R$. Total RNA was extracted from A549 and NCI-H1975 cells using TRIzol ${ }^{\circledR}$ reagent (Invitrogen; Thermo Fisher Scientific Inc.). A total of $2 \mu \mathrm{g}$ RNA was reverse transcribed into cDNA using PrimeScript RT reagent kit (Takara Bio Inc.) or HyperScript III miRNA 1st Strand cDNA Synthesis kit (Nova Biomedical) according to the manufacturer's protocol. Biomarker $2 \mathrm{X}$ SYBR-Green Fast qPCR Mix kit (Biomarker Technologies, Inc.) was used to assess the expression of indicated genes using the following thermocycling conditions: Initial denaturation at $95^{\circ} \mathrm{C}$ for $30 \mathrm{sec}$, followed by 50 cycles at $95^{\circ} \mathrm{C}$ for $5 \mathrm{sec}$ and $60^{\circ} \mathrm{C}$ for $30 \mathrm{sec}$ for annealing and elongation. Data were analyzed with $2^{-\Delta \Delta \mathrm{Cq}}$ method (18) with normalization to the gene expression levels of U6 and GAPDH. GAPDH forward, 5'-TACTAGCGGTTTTACGGGCG-3' and reverse, 5'-GAA CAGGAGGAGCAGAGAGCGA-3'; U6 forward, 5'-CTCGCT TCGGCAGCACA-3' and reverse 5'-AACGCTTCACGAATT TGCGT-3'; and PLOD1 forward primer,5'-AAGCCGGAGGAC AACCTTTT-3' and reverse 5'-AGCCTGTTTCGAAGGTC CAG-3'.

Overexpression of PLOD1 in A549 cells. PLOD1 cDNA was amplified from A549 cells via reverse transcription PCR with the following primers: PLOD1 forward, 5' TTGCGGCCGCA ACTTCACCTGGGGAGAGACTTC3' and reverse, 3' AGG ACTAAAAGGTtGTCTGAACCCGGATCCG5'. The amplified product was inserted into the pHAGE Puro 6tag lentiviral vector (Addgene, Inc.) with flags at the 5'-NotI-BamHI-3' fragments, referred to as the flag-tagged PLOD1 vector. To generate PLOD1-expressing lentiviruses, $293 \mathrm{~T}$ cells $\left(4 \times 10^{6} /\right.$ well) were co-transfected with $5 \mu \mathrm{g}$ flag-tagged PLOD1 lentiviral plasmid or the empty vector, and $10 \mu \mathrm{g}$ of two ad-helper plasmids (PSPAX2 and pMD2; Addgene, Inc.) using a calcium phosphate transfection kit (Shenzhen Biowit Biotech Co., Ltd.) at $37^{\circ} \mathrm{C}$. At $48 \mathrm{~h}$ post-transfection, the lentivirus-containing supernatants were obtained and subjected to ultracentrifugation through a $20 \%$ sucrose cushion at $1,300 \mathrm{x}$ g for $10 \mathrm{~min}\left(4^{\circ} \mathrm{C}\right)$. The harvested lentiviral particles were used to infect A549 cells at $50 \%$ confluence at an MOI of 0.1 , for $48 \mathrm{~h}$ at $37^{\circ} \mathrm{C}$. The following day, targeted A549 cells were screened with $2 \mu \mathrm{g} / \mathrm{ml}$ puromycin. After culturing for 8 days, the puromycin-resistant clones were obtained, and western blotting was performed to confirm PLOD1 expression. The empty pHAGE Puro 6tag lentiviral vector was used as a control.

Knockout of PLODI in NCI-H1975 and A549 cells. The CRISPR-Cas9 system was used to deplete PLOD1 expression in A549 and NCI-H1975 cells. Briefly, a single-guide RNA (sgRNA) sequence (5'-CTTGGCGTCGCCCTTCGCTTCG G-3') targeting exon 1 of the PLOD1 gene, and a non-targeting sgRNA (WT), were designed online (https://zlab. bio/guide-design-resources), synthesized by Sangon Biotech Co., Ltd., and then assembled into the CRISPR/Cas9 Lenti-V2 vector (Addgene, Inc.). Then, PLOD1 sgRNA CRISPR/Cas9 or its untargeted control were introduced into 293T cells ( 4 ×10\% $/$ well) using FuGeneHD transfection reagent (Promega Corporation) per the manufacturer's instructions, at room temperature for $24 \mathrm{~h}$. The resulting lentiviral particles were used to transduce A549 cells or NCI-H1975 cells at 55-65\% confluence for $48 \mathrm{~h}$, using Lipofectamine ${ }^{\circledR} 3000$ reagent (Invitrogen; Thermo Fisher Scientific, Inc.). The cells were treated with $2 \mu \mathrm{g} / \mathrm{ml}$ puromycin for 3 days to obtain stable cell lines (as aforementioned). Deletion of endogenous PLOD1 (knockout-1 and knockout-2 mutants) were selected from the pool of PLOD1-knockout cells by western blotting, and used in subsequent assays.

Cell Counting Kit 8 (CCK-8) assay. A total of $5 \times 10^{3}$ cells/well were cultured in 96 -well plates at $37^{\circ} \mathrm{C}$ for 24,48 and $72 \mathrm{~h}$. After a further 4 -h incubation with $10 \mu \mathrm{l}$ CCK-8 reagent according to the manufacturer's protocol, the cells were analyzed using a microplate reader, and the optical density value was recorded at $452 \mathrm{~nm}$.

Colony formation assay. A549 cells (500/well) were incubated for 2 weeks at $37^{\circ} \mathrm{C}$ in a $5 \% \mathrm{CO}_{2}$ incubator. After colony formation, the cells were subjected to 20 min of fixation with $4 \%$ paraformaldehyde at room temperature and $15 \mathrm{~min}$ of staining with $1 \%$ crystal violet at room temperature. Colonies with $\geq 50$ cells were counted an inverted light microscope (magnification, x200) (CKX41; Olympus Corporation) and plotted.

Western blotting. A549 cells were lysed in SDS lysis buffer at $95^{\circ} \mathrm{C}$ for $10 \mathrm{~min}$. Protein concentrations were determined 

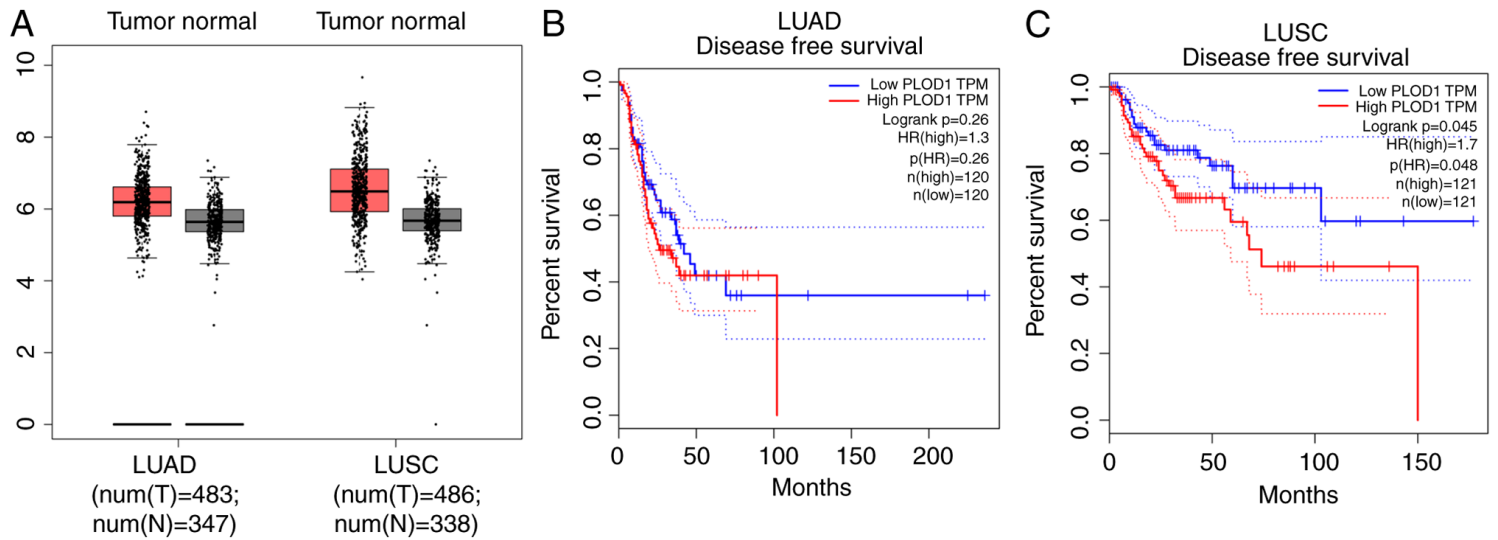

Figure 1. Expression of PLOD1 and correlation with patient prognosis in LUAD and LUSC. Data were collected from the GEPIA database. (A) PLOD1 expression in 1654 lung cancer tissues was compared with that in normal tissues using the GEPIA website. Kaplan-Meier analysis of (B) overall and (C) disease-free survival comparisons based on high and low PLOD1 expression levels in 240 LUAD and 242 LUSC cancer tissues, respectively, using Kaplan-Meier survival analysis. PLOD1, procollagen-lysine, 2-oxoglutarate 5-dioxygenase 1; LUAD, lung adenocarcinoma; LUSC, lung squamous cell carcinoma; GEPIA, Gene Expression Profiling Interactive Analysis.

using a BCA protein assay kit, and $20 \mu \mathrm{g}$ protein was resolved by $10 \%$ SDS-PAGE and electrotransferred onto PVDF membranes. After blocking with $5 \%$ semi-skimmed milk at room temperature for $30 \mathrm{~min}$, the membranes were probed with anti-flag (1:1,000; cat. no. F7425; Merck \& Co., Inc.), anti-PLOD1 (1:1,000; cat. no. K005553P; Shanghai Jinpan Biological Technology Co., Ltd.) or anti-GAPDH antibodies (1:1,000; cat. no. SAB2108668; Merck \& Co., Inc.) overnight at $4^{\circ} \mathrm{C}$, followed by incubation with an HRP-conjugated secondary antibody at room temperature for $1 \mathrm{~h}$. Finally, the blots were visualized using the Immobilon Western Chemiluminescent HRP Substrate kit (Merck KGaA).

Luciferase reporter assay. The E2F1 luciferase reporter plasmid (E2F1-Lu), which contains multiple E2F1 binding sites in its multiple cloning site, was purchased from Shanghai Yeasen Biotechnology Co., Ltd. E2F1 transcriptional activity was measured upon PLOD1 treatment with a Dual Luciferase Reporter Assay kit (Promega Corporation). Briefly, 293T cells were co-transfected with E2F1-Lu or pRL-TK plasmids and the flag-tagged PLOD1 vector (0, 100, 200 and $400 \mathrm{ng}$ each) using Lipofectamine ${ }^{\circledR} 3000$ reagent (Invitrogen; Thermo Fisher Scientific, Inc.) for 48 h. The Dual Luciferase Reporter Assay kit was adopted to detect luciferase activity. Renilla luciferase, expressed by the pRL-TK plasmid, was used to normalize firefly luciferase expression.

Bioinformatics analysis. PLOD1 expression data for 483 LUAD (lung adenocarcinoma), 486 LUSC (lung squamous cell carcinoma) were acquired and analyzed using the interactive web server GEPIA website (http://gepia. cancer-pku.cn/) according to the website instructions. Using the 'Co-expression' tab from the cBioPortal, Spearman's analysis was used determine the association between PLOD1 and H2AFX mRNA expression, which is reported as a critical downstream factor of PLOD1 (19). The criteria used for significant expression correlation between two genes was a Pearson's correlation coefficient $r \geq 0.3$ or $\leq-0.3$, a Spearman's correlation coefficient $r \geq 0.4$ or $\leq-0.4$, and all corresponding P-values $<0.05$.
Statistical analysis. Statistical analyses were performed using GraphPad Prism software 9. Significant differences between two groups were evaluated using an unpaired, two-tailed Student's t-test. For multiple comparisons, one-way ANOVA with Tukey's post hoc test was employed. $\mathrm{P}<0.05$ was considered to indicate a statistically significant difference.

\section{Results}

PLOD1 upregulation in LUAD and LUSC and its prognostic value in lung cancer. First, the PLOD1 expression levels in LUAD and LUSC were analyzed in a lung cancer cohort from the GEPIA dataset. GEPIA datasets comprising 483 LUAD and 486 LUSC samples were used in the present study. As shown in Fig. 1A, PLOD1 mRNA expression was increased in both subtypes of lung cancer compared with the corresponding normal tissues. Using the GEPIA data, disease-free survival (DFS) was also assessed by PLOD1 quartiles using the Kaplan-Meier method (log-rank test) (Fig. 1B). For patients with LUSC, higher PLOD1 expression resulted in shorter DFS $(\mathrm{P}=0.048)$ although no significant difference was observed in patients with LUAD. These findings indicated that the upregulation of PLOD1 may influence the malignancy of LUSC.

Ectopic expression of PLODI enhances the proliferation and colony formation capacity of A549 cells. Next, PLOD1 was overexpressed in A549 cells, as verified by western blotting (Fig. 2A). A CCK-8 assay was performed to assess A549 cell proliferative capacity. The results demonstrated that the proliferation rate of A549 cells overexpressing PLOD1 was significantly improved after 5 days (Fig. 2B). Coincidentally, colony formation assays revealed that the upregulation of PLOD1 enhanced the clonogenic capacity of A549 cells (Fig. 2C and D), which is consistent with the results of the CCK8 assay. These data suggested that PLOD1-overexpression promoted the proliferation and colony formation ability of A549 cells.

Targeted depletion of PLOD1 suppresses the proliferation of lung cancer cells. CRISPR/Cas9-mediated knockout of PLOD1 

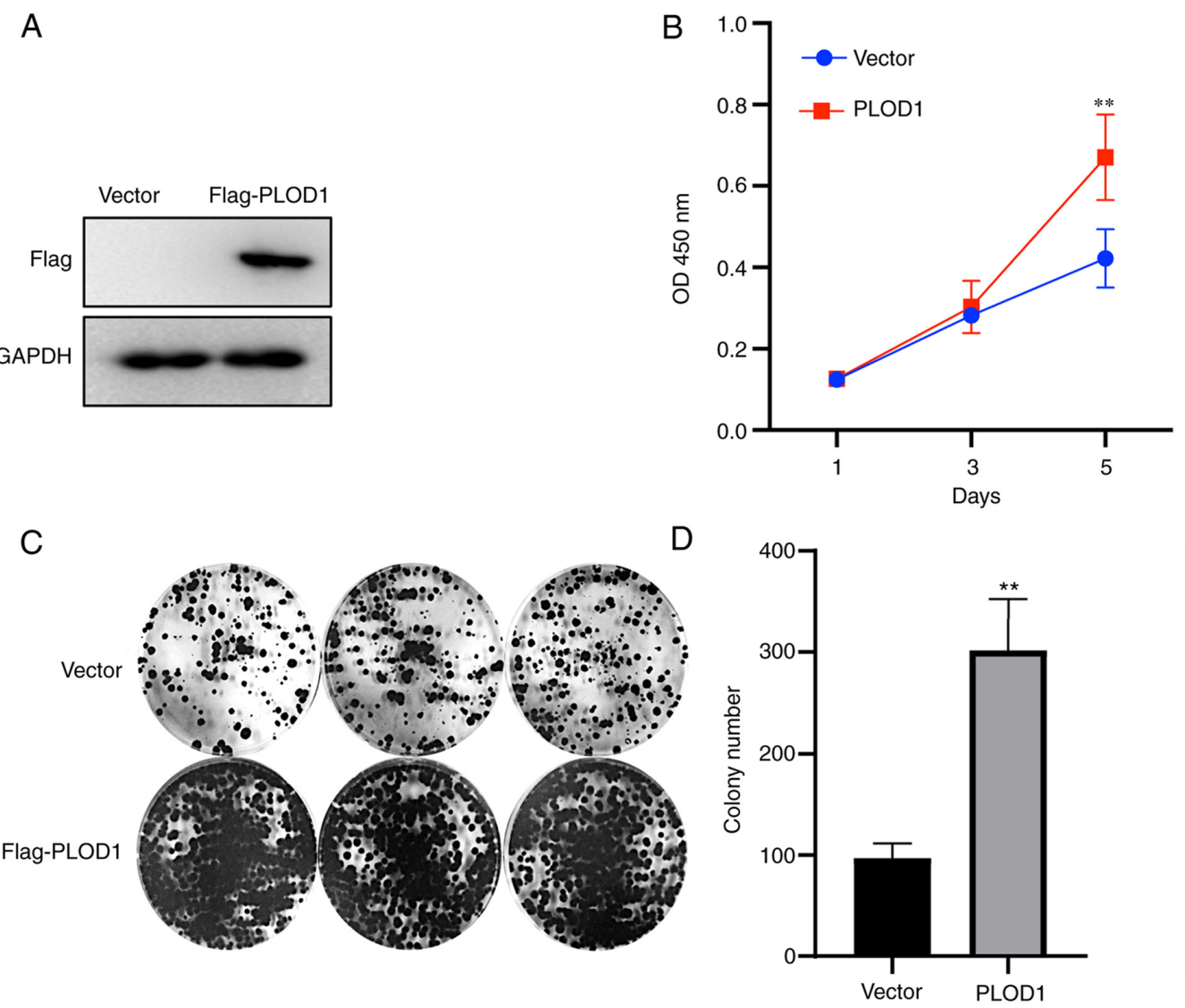

Figure 2. Overexpression of PLOD1 promotes cellular proliferation and colony formation. (A) PLOD1 protein expression levels in A549 cells were assessed by western blot analysis using GAPDH as the internal control. (B) Cellular proliferation was assessed using Cell Counting Kit 8 assays at 1,3 , and 5 days of culture. (C and D) Effects of PLOD1 on the proliferation of A549 cells were assessed by cell colony formation capacity. ** P<0.01. PLOD1, procollagen-lysine, 2-oxoglutarate 5-dioxygenase 1.

was subsequently performed in A549 and NCI-H1975 cells (Figs. 3A and 4A). The CCK-8 assay showed that cellular proliferation was significantly decreased following PLOD1-knockout (Figs. 3B and 4B). Furthermore, the colony formation capacity of PLOD1-deficient A549 cells and NCI-H1975 cells was attenuated compared with that of the control cells (Figs. 3C and D and 4C and D). These data indicated that PLOD1 serves an important role in lung cancer development.

PLOD1 regulates E2F1 pathway activation in lung cancer development. Because E2F1 is reported to play an oncogenic role in lung cancer (15), the regulatory relationship between PLOD1 and E2F1 was investigated using a luciferase reporter assay. As presented in Fig. 5A, the transcriptional activity of E2F1 was increased in a PLOD1-dependent manner. In particular, a 5-fold increase in transcriptional activity was observed in 293T cells treated with $400 \mathrm{ng}$ of PLOD1. H2AFX is a predominant sensor protein that is regulated by ectopic expression of E2F1, and is involved in DNA repair and cell survival (20). Therefore, the correlation between PLOD1 and $\mathrm{H} 2 \mathrm{AFX}$ expression was also analyzed to further reveal the mechanism of PLOD1/E2F1-mediated lung cancer tumorigenesis. The results of Spearman's analysis showed that PLOD1 mRNA expression was positively correlated with that of H2AFX mRNA (Fig. 5B). These results indicated that PLOD1 may be involved in the E2F1 pathway.

\section{Discussion}

PLOD1 belongs to the PLOD family, which is responsible for manipulating collagen crosslink formation and maturation. Although PLOD2 and PLOD3 have been well investigated in tumors $(21,22)$, the function of PLOD1 in tumors, including lung cancer, remains to be elucidated. In a multicohort, retrospective study of patients with LUAD, high PLOD1 expression levels were associated with an unfavorable prognosis (8). The findings of the present study highlight that PLOD1 plays a tumor-promoting role in lung cancer. First, PLOD1 was revealed to be highly expressed in LUAD and LUSC samples compared with normal lung tissues, and its increased expression was also associated with poor prognosis in patients with lung cancer. Secondly, elevated expression of PLOD1 considerably increased the proliferative and colony formation capacity of A549 cells, while PLOD1-knockout nakedly reduced proliferative ability and cell viability. Finally, investigation of the molecular mechanism indicated that the PLOD1-E2F1 axis serves a pro-tumorigenic role in lung cancer.

Enhanced collagen deposition and crosslinking is a typical characteristic of extracellular matrix remodeling during neoplastic transformation and tumor progression, indicating that PLOD1 plays a vital role in multistep carcinogenesis (6). Thus in the present study, the expression status of PLOD1 in lung cancer was investigated. PLOD1 mRNA levels were 
A

A549

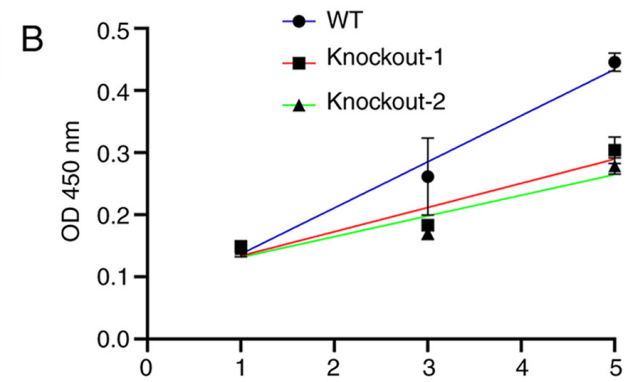

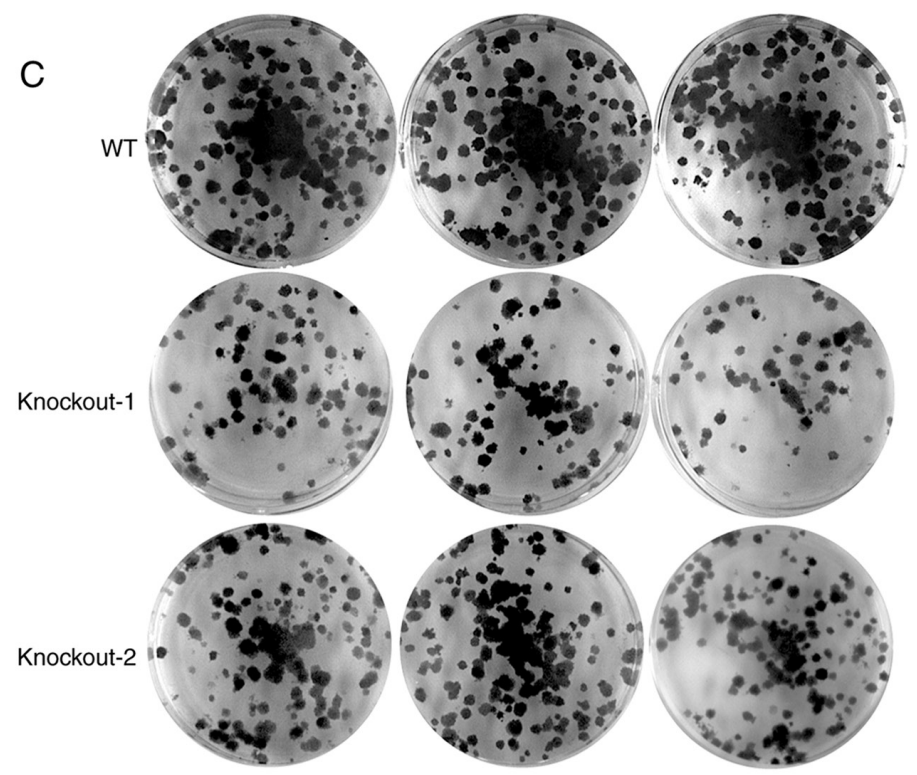

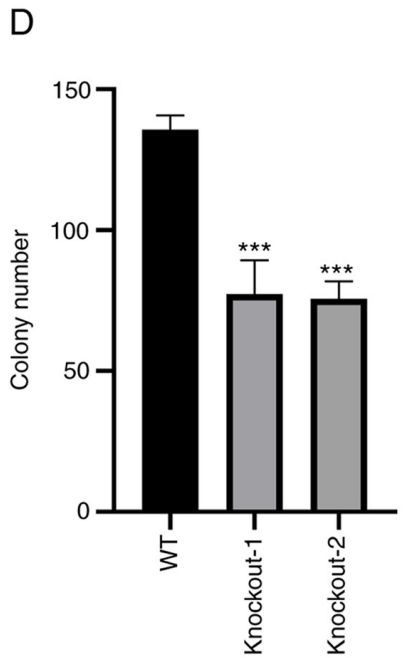

Figure 3. PLOD1-knockout using the CRISPR/Cas9 system suppressed the proliferation of A549 cells. (A) PLOD1 protein expression levels in A549 cells was assessed by western blot analysis using GAPDH as the internal control. (B) Cellular proliferation was assessed by MTT assay at 1, 3, and 5 days of culture. (C and D) Effects of PLOD1 on the proliferation of A549 cells were assessed by cell colony formation capacity. ${ }^{* * *} \mathrm{P}<0.001$. PLOD1, procollagen-lysine, 2-oxoglutarate 5-dioxygenase 1; WT, wild-type.

significantly upregulated in both LUAD and LUSC samples compared with normal lung samples. Moreover, GEPIA database analysis revealed a negative correlation between PLOD1 expression and patient prognosis.

Considering that tumor-sustaining proliferative capacity is a critical hallmark of cancer development, including in lung cancer (23), the role of PLOD1 in the proliferation and colony formation of A549 and NCI-H1975 cells was investigated. Unsurprisingly, the results demonstrated that PLOD1 significantly increased the proliferative and colony formation capacity of A549 lung cancer cells. The results also indicated that PLOD1, a proliferation promoter, may also stimulate lung cancer cell proliferation and thus facilitate lung cancer malignancy. The tumor-promoting activity of PLOD1 has also been observed in other malignant cancers $(11,24)$. For instance, PLOD1 caused abnormalities in the Hippo signaling pathway and contributed to the increased proliferative and invasive capacities of osteosarcoma cells (12).

Currently, multiple lines of evidence indicate that E2F1 serves as a multifunctional oncogenic factor in lung cancer $(16,25)$. For example, E2F1 silencing in two small-cell lung cancer cell lines (H446 and H1688) considerably suppressed epithelial-mesenchymal transition (25). E2F1 also increased the invasive, migratory and metastatic capacity of small-cell lung cancer cell lines by interacting with several cytokines, such as the matrix metalloproteinases ankyrin repeat domain-containing protein 22 and PTEN $(15,26)$. A high expression level of E2F1 also serves as an unfavorable prognostic variable, as well as a tumor staging benchmark, in patients with lung cancer (16). Additionally, E2F1 has been shown to be a critical downstream molecule of CDK4 and promotes the proliferation and survival of non-small-cell lung cancer cell lines (27). In the present study, luciferase reporter assays showed that PLOD1 activated E2F1 transcriptional activity, indicating that it may influence cell proliferation and colony formation by upregulating E2F1. The positive association between PLOD1 and H2AFX further supported the regulatory relationship between PLOD1 and E2F1. Regarding the PLOD1/E2F1 axis, E2F1 was also found to bind to the PLOD1 promoter and induce its expression in human embryonic lung fibroblast cell lines (2BS and WI-38 cells) (28), which may indicate a positive regulation loop between PLOD1 and E2F1. Furthermore, PLOD1 and E2F1 are reported to be two critical downstream regulatory factors of $\mathrm{NF}-\kappa \mathrm{B}(29,30)$. These findings support the regulatory relationship of E2F1 and PLOD1 observed in the present study. Further mechanistic studies will focus on the complex regulatory network of PLOD1. 
A
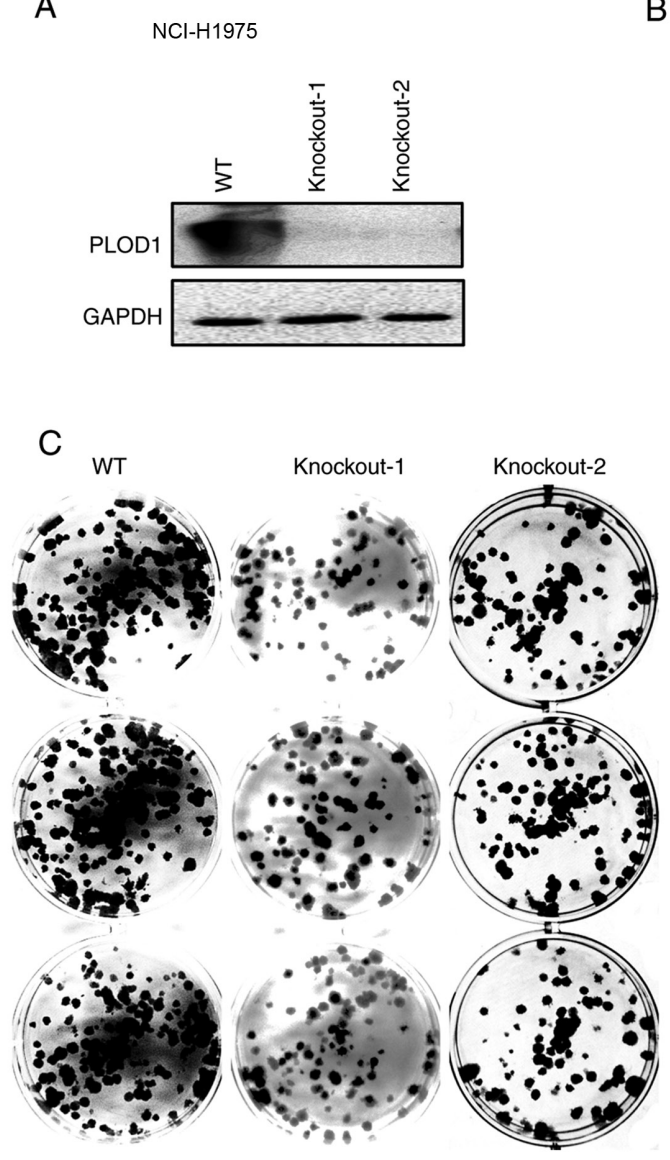
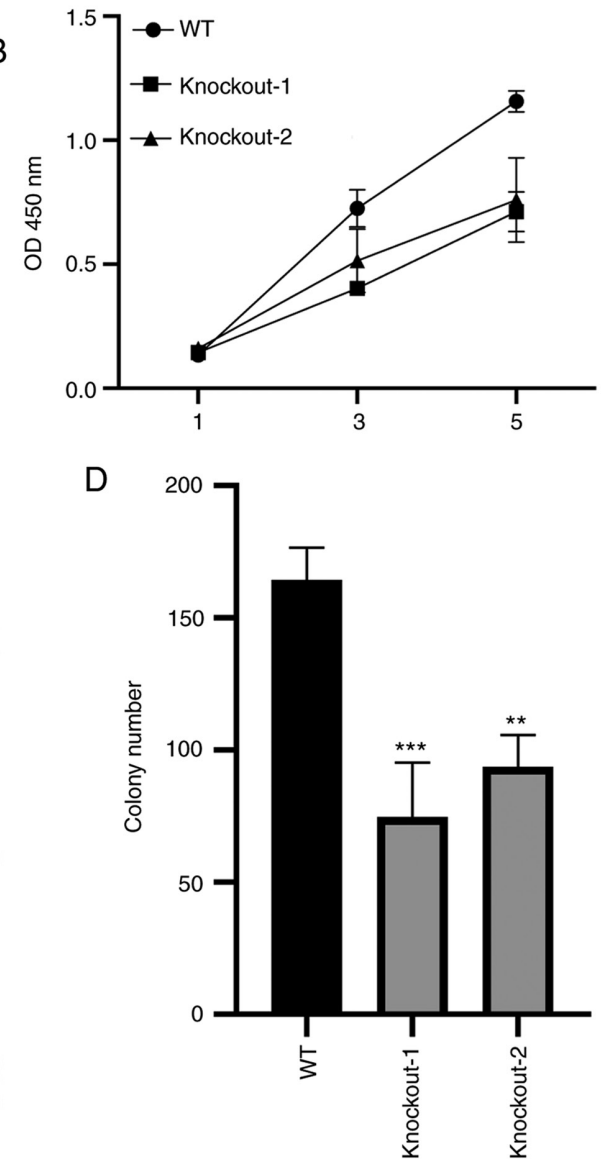

Figure 4. PLOD1-knockout using the CRISPR/Cas9 system suppressed the proliferation of NCI-H1975 cells. (A) Western blot assays were conducted to verify the knockout efficiency of the PLOD1 in NCI-H1975 cells. (B) Cell Counting Kit 8 assays were used to assess the effects of PLOD-knockout on the proliferation of NCI-H1975 cells. (C and D) Effects of PLOD1 on the proliferation of NCI-H1975 cells were measured using cell colony formation assays. ${ }^{* *} \mathrm{P}<0.01$ and ${ }^{* * *} \mathrm{P}<0.001$. PLOD1, procollagen-lysine, 2-oxoglutarate 5-dioxygenase 1; WT, wild-type.
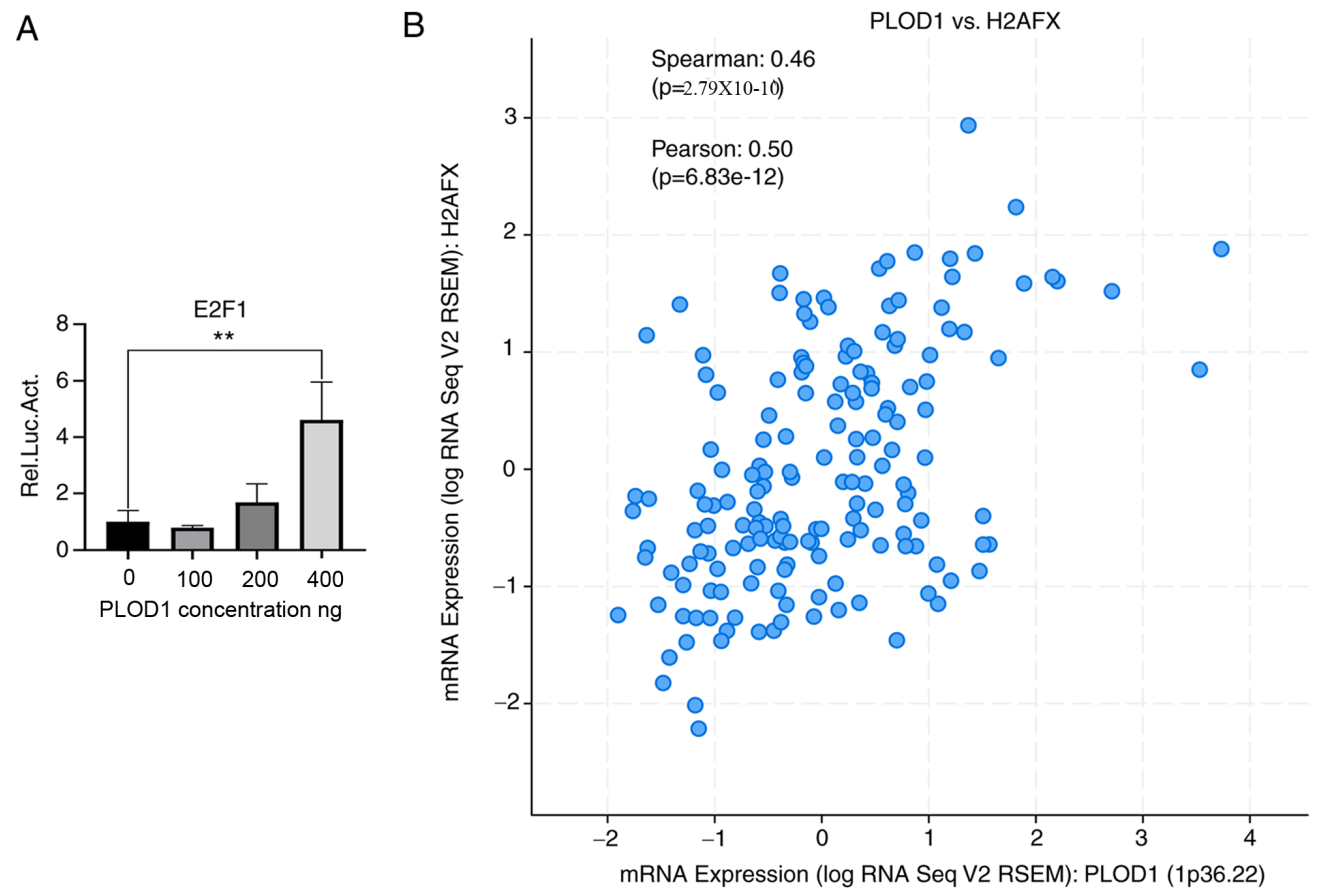

Figure 5. Regulatory relationship between PLOD1 and E2F1 in lung cancer. (A) Interaction between E2F1 and PLOD1 was determined by luciferase reporter assay. A549 cells were transfected with different dose of flag-tagged PLOD1 lentiviral plasmids. (B) Correlation analysis of PLOD1 and H2AFX mRNA expression using cBioPortal by Spearman's analysis; Pearson's correlation, 0.50; Spearman's correlation, 0.46. * P $<0.01$. PLOD1, procollagen-lysine, 2-oxoglutarate 5-dioxygenase 1; E2F1, E2F transcription factor 1; H2AFX, H2A histone family member X. 
Taken together, the results of the present study demonstrated that PLOD1 mRNA expression was increased in both LUAD and LUSC and promoted lung cancer cell proliferation and colony formation by activating E2F1 expression. These findings indicate that regulation of the PLOD1/E2F1 axis represents a potential therapeutic strategy for patients with lung cancer.

\section{Acknowledgements}

Not applicable.

\section{Funding}

The present study was supported by the National Natural Science Foundation of China (grant no. 81960026), the Key Research and Development Program of Guangxi Science \& Technology Department (grant no. Gui AB16380218), the Guangxi Natural Science Foundation (grant no. Gui.0728081), the Self-raised project of Guangxi Health Commission (grant. no. Z20201117) and the Guangxi Natural Science Foundation Project, China (grant no. 2016GXNSFAA380196).

\section{Availability of data and materials}

The datasets generated and/or analyzed during the current study are available in the [GEPIA] repository, [http://gepia. cancer-pku.cn/index.html].

\section{Author's contributions}

BJL and HY performed the experiments, analyzed the data and wrote the manuscript. BJL and HY confirmed the authenticity of the data. BS, JWH and ZQQ were responsible for the bioinformatics information, analysis and interpretation of data. All authors have read and approved the final manuscript.

\section{Ethics approval and consent to participate}

Not applicable.

\section{Patient consent for publication}

Not applicable.

\section{Competing interests}

The authors declare that they have no competing interests.

\section{References}

1. Bray F, Ferlay J, Soerjomataram I, Siegel RL, Torre LA and Jemal A: Global cancer statistics 2018: GLOBOCAN estimates of incidence and mortality worldwide for 36 cancers in 185 countries. CA Cancer J Clin 68: 394-424, 2018. Erratum in: CA Cancer J Clin 70: 313, 2020.

2. International Agency for Research on Cancer (IARC): World Cancer Report: Cancer Research for Cancer Prevention. Wild CP, Weiderpass E and Stewart BW (eds). IARC, Lyon, 2020.

3. Koudelakova V, Kneblova M, Trojanec R, Drabek J and Hajduch M: Non-small cell lung cancer - genetic predictors. Biomed Pap Med Fac Univ Palacky Olomouc Czech Repub 157: 125-136, 2013.
4. Clausen MM and Langer SW: Improving the prognosis for lung cancer patients. Acta Oncol 58: 1077-1078, 2019.

5. Woodard GA, Jones KD and Jablons DM: Lung cancer staging and prognosis. Cancer Treat Res 170: 47-75, 2016.

6. Qi Y and Xu R: Roles of PLODs in collagen synthesis and cancer progression. Front Cell Dev Biol 6: 66, 2018.

7. Eisinger-Mathason TS, Zhang M, Qiu Q, Skuli N, Nakazawa MS Karakasheva T, Mucaj V, Shay JE, Stangenberg L, Sadri N, et al: Hypoxia-dependent modification of collagen networks promotes sarcoma metastasis. Cancer Discov 3: 1190-1205, 2013.

8. Chang WH, Forde D and Lai AG: Dual prognostic role of 2-oxoglutarate-dependent oxygenases in ten cancer types: Implications for cell cycle regulation and cell adhesion maintenance. Cancer Commun (Lond) 39: 23, 2019.

9. LiSS, Lian YF, Huang YL, Huang YH and Xiao J: Overexpressing PLOD family genes predict poor prognosis in gastric cancer. J Cancer 11: 121-131, 2020.

10. Zhang T, Li Y, Zhu R, Song P, Wei Y, Liang T and Xu G: Transcription factor $\mathrm{p} 53$ suppresses tumor growth by prompting pyroptosis in non-small-cell lung cancer. Oxid Med Cell Longev 2019: 8746895, 2019.

11. Yamada Y, Kato M, Arai T, Sanada H, Uchida A, Misono S, Sakamoto S, Komiya A, Ichikawa T and Seki N: Aberrantly expressed PLOD1 promotes cancer aggressiveness in bladder cancer: A potential prognostic marker and therapeutic target. Mol Oncol 13: 1898-1912, 2019.

12. Wu X, Xiang H, Cong W, Yang H, Zhang G, Wang Y, Guo Z, Shen Y and Chen B: PLOD1, a target of miR-34c, contributes to cell growth and metastasis via repressing LATS1 phosphorylation and inactivating Hippo pathway in osteosarcoma. Biochem Biophys Res Commun 527: 29-36, 2020.

13. Hollern DP, Honeysett J, Cardiff RD and Andrechek ER: The E2F transcription factors regulate tumor development and metastasis in a mouse model of metastatic breast cancer. Mol Cell Biol 34: 3229-3243, 2014.

14. Denechaud PD, Fajas L and Giralt A: E2F1, a novel regulator of metabolism. Front Endocrinol (Lausanne) 8: 311, 2017.

15. Malaney P, Palumbo E, Semidey-Hurtado J, Hardee J, Stanford K, Kathiriya JJ, Patel D, Tian Z, Allen-Gipson D and Davé V: PTEN physically interacts with and regulates E2F1-mediated transcription in lung cancer. Cell Cycle 17: 947-962, 2018.

16. Sun CC, Zhou Q, Hu W, Li SJ, Zhang F, Chen ZL, Li G, Bi ZY, Bi YY, Gong FY, et al: Transcriptional E2F1/2/5/8 as potential targets and transcriptional E2F3/6/7 as new biomarkers for the prognosis of human lung carcinoma. Aging (Albany NY) 10: 973-987, 2018

17. Zhao M, Liu Y, Chang J, Qi J, Liu R, Hou Y, Wang Y, Zhang X, Qiao L and Ren L: ILF2 cooperates with E2F1 to maintain mitochondrial homeostasis and promote small cell lung cancer progression. Cancer Biol Med 16: 771-783, 2019.

18. Livak KJ and Schmittgen TD: Analysis of relative gene expression data using real-time quantitative PCR and the 2(-Delta Delta C(T)) Method. Methods 25: 402-408, 2001.

19. Liu J, Li W, Zhang J, Ma Z, Wu X and Tang L: Identification of key genes and long non-coding RNA associated ceRNA networks in hepatocellular carcinoma. PeerJ 7: e8021, 2019.

20. Folk WP, Kumari A, Iwasaki T, Pyndiah S, Johnson JC, Cassimere EK, Abdulovic-Cui AL and Sakamuro D: Loss of the tumor suppressor BIN1 enables ATM Ser/Thr kinase activation by the nuclear protein E2F1 and renders cancer cells resistant to cisplatin. J Biol Chem 294: 5700-5719, 2019.

21. Du H, Pang M, Hou X, Yuan S and Sun L: PLOD2 in cancer research. Biomed Pharmacother 90: 670-676, 2017.

22. He J, Wei W, Yang Q and Wang Y: Phillygenin exerts in vitro and in vivo antitumor effects in drug-resistant human esophageal cancer cells by inducing mitochondrial-mediated apoptosis, ROS generation, and inhibition of the nuclear factor kappa B NF- $\kappa \mathrm{B}$ signalling pathway. Med Sci Monit 25: 739-745, 2019.

23. Hanahan D and Weinberg RA: Hallmarks of cancer: The next generation. Cell 144: 646-674, 2011.

24. Wang D, Zhang S and Chen F: High expression of PLOD1 drives tumorigenesis and affects clinical outcome in gastrointestinal carcinoma. Genet Test Mol Biomarkers 22: 366-373, 2018.

25. Allan RJ and Roxon JJ: The effect of dioctyl sodium sulphosuccinate on tartrazine azo reduction by intestinal bacteria. Xenobiotica 7: 181-186, 1977.

26. Yin J, Fu W, Dai L, Jiang Z, Liao H, Chen W, Pan L and Zhao J: ANKRD22 promotes progression of non-small cell lung cancer through transcriptional up-regulation of E2F1. Sci Rep 7: 4430, 2017. 
27. Wu B and Liu R: PAQR4 promotes cell proliferation and metastasis through the CDK4-pRB-E2F1 pathway in non-small-cell lung cancer. OncoTargets Ther 12: 3625-3633, 2019.

28. Gao S, Song Q, Liu J, Zhang X, Ji X and Wang P: E2F1 mediates the downregulation of POLD1 in replicative senescence. Cell Mol Life Sci 76: 2833-2850, 2019.

29. Palomer X, Álvarez-Guardia D, Davidson MM, Chan TO, Feldman AM and Vázquez-Carrera M: The interplay between NF-kappaB and E2F1 coordinately regulates inflammation and metabolism in human cardiac cells. PLoS One 6: e19724, 2011.
30. Mia MM and Bank RA: The IкB kinase inhibitor ACHP strongly attenuates TGF $\beta 1$-induced myofibroblast formation and collagen synthesis. J Cell Mol Med 19: 2780-2792, 2015.

cc) (i) $($ This work is licensed under a Creative Commons

EY NG ND Attribution-NonCommercial-NoDerivatives 4.0 International (CC BY-NC-ND 4.0) License. 